\title{
FORMAL AND CONVERGENT POWER SERIES SOLUTIONS OF SINGULAR PARTIAL DIFFERENTIAL EQUATIONS
}

\author{
BY
}

\author{
STANLEY KAPLAN
}

\begin{abstract}
ABSTRACr. A class of singular first-order partial differential equations is described for which an analogue of a theorem of $\mathbf{M}$. Artin on the solutions of analytic equations holds: given any formal power series solution and any nonnegative integer $\nu$, a convergent power series solution may be found which agrees with the given formal solution up to all terms of order $\langle\nu$.
\end{abstract}

\section{Introduction. Let}

$$
X=\sum_{i=1}^{d} X_{i}(z) \frac{\partial}{\partial z_{i}}
$$

be a first-order linear differential operator with coefficients $X_{i}(z)$ which are power series in the $d$ variables $z=\left(z_{1}, z_{2}, \ldots, z_{d}\right)$, convergent in some neighborhood of the origin $\mathbf{0}$ in $\mathbf{C}^{d}$. (For short, we write $X_{i}(z) \in \mathbf{C}\{z\}$, $i=1, \ldots, d$.) We seek $p$-tuples $u(z)=\left(u_{1}(z), \ldots, u_{p}(z)\right)$ of formal power series with no constant term which satisfy systems of equations of the form

$$
X u_{i}(z)=f_{i}(z, u(z)), \quad i=1, \ldots, p,
$$

where each $f_{i}(z, u)$ is a convergent power series in $\left(z_{1}, \ldots, z_{d}, u_{1}, \ldots, u_{p}\right)$. (We write $\left.u(z) \in[C[z]]_{0}\right]^{p}$, the subscript 0 denoting constant term zero, and $f(z, u)=\left(f_{i}(z, u), \ldots, f_{p}(z, u)\right) \in[\mathbf{C}\{z, u\}\}^{p}$.) We assume that $X$ is singular, in the sense that $X_{i}(0)=0, i=1, \ldots, d$; in the nonsingular case the problem of the existence and convergence of power series solutions of (1) is easily treated by methods which go back to Cauchy. In fact, if $X(0)=$ $\left(X_{1}(0), \ldots, X_{d}(0)\right) \neq 0$, let $S$ be any $d-1$ dimensional analytic submanifold through 0 in $\mathbf{C}^{d}$, transverse at 0 to $X(0)$. Consideration of the characteristic equation $\dot{z}=X(z)$ leads to a change of variables $\zeta=\zeta(z)$ (by which we mean a $d$-tuple $\zeta(z)=\left(\zeta_{1}(z), \ldots, \zeta_{d}(z)\right)$ of elements of $\mathbf{C}\{z\}$ such that the $d \times d$ matrix $\left(\partial \zeta_{i}(0) / \partial z_{j}\right)_{i, j=1, \ldots, d}$ is invertible and where $z=z(\zeta)$ is used to denote the inverse change of variables) with the following properties:

(i) $S=\left\{z \in \mathbf{C}^{d}: \zeta_{d}(z)=0\right\}$ near 0 in $\mathbf{C}^{d}$; and

(ii) for any $y(z) \in \mathbf{C}[z]]$,

$$
X y(z(\zeta))=\frac{\partial}{\partial \zeta_{d}}\{y(z(\zeta))\}
$$

Received by the editors May 19, 1978 and, in revised form, October 19, 1978.

AMS (MOS) subject classifications (1970). Primary 35C10, 35A35; Secondary 35F20, $35 \mathrm{~A} 10$. 
(we say, for short, that the change of variables $\zeta=\zeta(z)$ transforms $X$ into $\left.\partial / \partial \zeta_{d}\right)$. We now work in the variables $\zeta$, and write $\zeta^{\prime}$ for $\left(\zeta_{1}, \zeta_{2}, \ldots, \zeta_{d-1}\right)$. For any $f(z, u) \in[\mathbf{C}\{z, u\}\}^{p}$ the following assertions are easily shown:

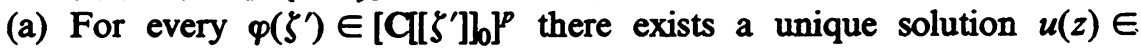
$\left.[\mathrm{C}[z]]_{0}\right]^{p}$ of $(1)$ with $u\left(z\left(\zeta^{\prime}, 0\right)\right)=\varphi\left(\zeta^{\prime}\right)$.

(b) With the same notation, $u(z) \in[\mathbf{C}\{z\}\}^{p}$ if and only if $\varphi\left(\zeta^{\prime}\right) \in\left[\mathbf{C}\left\{\zeta^{\prime}\right\}\right\}^{p}$.

(c) If $u^{*}(z)$ and $\varphi^{*}\left(\zeta^{\prime}\right)$ are related as $u(z)$ and $\varphi\left(\zeta^{\prime}\right)$ above, then

$$
\operatorname{ord}\left\{u(z)-u^{*}(z)\right\}=\operatorname{ord}\left\{\varphi\left(\zeta^{\prime}\right)-\varphi^{*}\left(\zeta^{\prime}\right)\right\}
$$

Here, we define

$$
\text { ord } u(z)=\min \left\{\text { ord } u_{i}(z): 1<i<p\right\}
$$

for $u(z)=\left(u_{1}(z), \ldots, u_{p}(z)\right)$. (Given $\left.y(z) \in \mathrm{q}[z]\right]$ with $y(z)=\Sigma y_{\alpha} z^{\alpha}$ where each $y_{\alpha} \in \mathbf{C}$, and the sum is taken over the set $g_{d}$ of all $d$-tuples $\alpha=$ $\left(\alpha_{1}, \ldots, \alpha_{d}\right)$ of nonnegative integers, we define ord $y(z)=\inf \left\{|\alpha|=\alpha_{1}+\alpha_{2}\right.$ $\left.+\cdots+\alpha_{d}: \alpha \in \mathscr{G}_{d}, y_{\alpha} \neq 0\right\}$.)

An easy consequence of (a), (b), and (c) is the following analogue of a theorem of M. Artin [1] on solutions of equations of the form

$$
f(z, u(z))=0
$$

(with the notation already used above):

(d) Suppose $X$ is nonsingular. If $\left.u(z) \in[\mathrm{C}[z]]_{0}\right]^{p}$ satisfies (1), then, given any positive integer $\nu$, there exists $\tilde{u}(z) \in\left[C\{z\}_{0}\right]^{p}$ such that $\tilde{u}(z)$ satisfies (1) as well, and $\operatorname{ord}(\tilde{u}(z)-u(z)) \geqslant \nu$.

To see that this no longer holds in general in the singular case, we recall an example due to Euler: with $d=p=1$, the equation

$$
z^{2} \frac{d}{d z} u(z)=u(z)-z
$$

has the unique power series solution

$$
u(z)=\sum_{k=1}^{\infty}(k-1) ! z^{k}
$$

which is not convergent. That this phenomenon is not limited to the case of one variable and that it does not depend entirely on the vanishing to second-order of the coefficients of $X$ is seen by consideration of the equation in two variables $z=\left(z_{1}, z_{2}\right)$ :

$$
\left(z_{1}+\frac{1}{2} z_{1}^{2} z_{2}\right) \frac{\partial}{\partial z_{1}} u(z)-\left(z_{2}-\frac{1}{2} z_{1} z_{2}^{2}\right) \frac{\partial}{\partial z_{2}} u(z)=u(z)-z_{1} z_{2}
$$

which has the unique power series solution

$$
u(z)=\sum_{k=1}^{\infty}(k-1) !\left(z_{1} z_{2}\right)^{k} .
$$

In fact, suppose $u(z) \in \mathrm{C}[z]]_{0}$, and write $u(z)=\Sigma_{\alpha \in \Phi_{2}} u_{\alpha} z^{\alpha}$, with $u_{0}=0$. (3) 
is equivalent to the infinite system of equations

$$
\left(\alpha_{1}-\alpha_{2}-1\right) u_{\alpha}+\frac{1}{2}\left(\alpha_{1}+\alpha_{2}\right) u_{\alpha^{\prime}}=\varepsilon_{\alpha} \quad\left(\alpha \in g_{2}\right)
$$

where we have written $\alpha^{\prime}$ to mean either $\left(\alpha_{1}-1, \alpha_{2}-1\right)$ if this is in $g_{2}$, or $0=(0,0) \in I_{2}$ otherwise, and $\varepsilon_{\alpha}=-1$ if $\alpha=(1,1), \varepsilon_{\alpha}=0$ in all other cases. From this we see at once that $u_{\alpha}=0$ unless $\alpha_{1}=\alpha_{2}$ and thus $u(z)$ is of the form

$$
u(z)=\varphi\left(z_{1} z_{2}\right) \quad \text { with } \varphi(t)=\sum_{k=1}^{\infty} \varphi_{k} t^{k} \in \mathbf{C}[[t]]_{0} .
$$

But, for (3) to hold in this case, it is necessary and sufficient that $t^{2} \varphi^{\prime}(t)=$ $\varphi(t)-t$, whence our assertion.

The purpose of the present work is to isolate a subclass of singular $X$ for which something like the theory described above in the nonsingular case still holds. Our hypotheses on $X$ concern the zero variety $S$ of the coefficients $X_{1}(z), \ldots, X_{d}(z)$ and the eigenvalues of the linear part of $X(z)=$ $\left(X_{1}(z), \ldots, X_{d}(z)\right)$ (see $\left(\mathrm{H}_{1}\right)$ and $\left(\mathrm{H}_{2}\right)$ below). In place of (a) we obtain a bijective correspondence between formal solutions of (1) and formal solutions of an associated system of equations of the form (2). These latter equations express compatibility conditions which must be satisfied by the "restriction to $S$ " of a solution of (1) and a certain number of its tangential derivatives. The main part of our work is to show that assertion (b) carries over in this case (i.e., convergent solutions of one system correspond to convergent solutions of the other). Assertion (c) also goes over (the correspondence preserves "order") so Artin's theorem cited above may be applied to give a version of (d) in our singular case.

2. The main results. We assume from here on that $X(0)=0$ and we denote by $S$ the germ at $0 \in \mathrm{C}^{d}$ of the analytic variety

$$
\left\{z \in \mathbf{C}^{d}: X_{i}(z)=0, i=1, \ldots, d\right\} .
$$

The following hypothesis on $X$ will be assumed to hold throughout the remainder of this work:

$\left(\mathrm{H}_{1}\right): S$ is the germ at $\mathbf{0} \in \mathrm{C}^{d}$ of an analytic submanifold of $\mathrm{C}^{d}$, of dimension $s$, where $0 \leqslant s \leqslant d-1$. We write $n=d-s$ throughout. Then $\left(\mathrm{H}_{1}\right)$ means: There exists a change of variables $\zeta=\zeta(z)$ such that

$$
S=\left\{z \in \mathbf{C}^{d}: \zeta_{1}(z)=\zeta_{2}(z)=\cdots=\zeta_{n}(z)=0\right\} \text { near } 0 .
$$

We say in this case that the variables $\zeta=\left(\zeta_{1}, \ldots, \zeta_{d}\right)$ are adapted to $S$ and we write them in the form $\zeta=(x, \xi)=\left(x_{1}, \ldots, x_{n}, \xi_{1}, \ldots, \xi_{s}\right)$ where $x_{i}=\zeta_{i}$, $i=1, \ldots, n$, and $\xi_{j}=\zeta_{n+j}$ for $j=1, \ldots, s$. Observe that any change of variables $\zeta=\zeta(z)$ (adapted to $S$ or not) transforms 


$$
X=\sum_{i=1}^{d} X_{i}(z) \frac{\partial}{\partial z_{i}} \quad \text { into } \quad \sum_{i=1}^{d} \tilde{X}_{i}(\zeta) \frac{\partial}{\partial \zeta_{i}}
$$

where, writing $X(z)$ and $\tilde{X}(\zeta)$ as column vectors and writing $\partial z(\zeta) / \partial \zeta$ for the $d \times d$ matrix $\left(\partial z_{i}(\zeta) / \partial \zeta_{j}\right)_{i, j=1, \ldots, d}$ we have

$$
X(z(\zeta))=\frac{\partial z}{\partial \zeta}(\zeta) \tilde{X}(\zeta)
$$

Since $X(0)=0$, we conclude from this that the $d \times d$ matrices $\partial X(0) / \partial z$ and $\partial \tilde{X}(0) / \partial \zeta$ are related by

$$
\frac{\partial X}{\partial z}(0)=\frac{\partial z}{\partial \zeta}(0) \frac{\partial \tilde{X}}{\partial \zeta}(0)\left(\frac{\partial z}{\partial \zeta}(0)\right)^{-1}
$$

As $\partial \tilde{X}(0) / \partial \zeta$ has an $s \times s$ block of zeros in its lower right-hand corner, we conclude further that the characteristic polynomial $P(\lambda)$ of $\partial X(0) / \partial z$ has the form $P(\lambda)=\lambda^{s} Q(\lambda)$, with $Q(\lambda)$ a monic polynomial of degree $n$.

Our second hypothesis goes back to Poincarés thesis [5, p. 100]; in the sequel we invoke it explicitly when needed:

$\left(\mathrm{H}_{2}\right)$ : Let $\lambda_{1}, \ldots, \lambda_{n}$ denote the zeros of $Q(\lambda)$, repeated according to algebraic multiplicity. Let $\Lambda$ denote the convex hull in $C$ of the set $\left\{\lambda_{1}, \ldots, \lambda_{n}\right\}$ (i.e., $\Lambda=\left\{\sum_{i=1}^{n} t_{i} \lambda_{i}: 0 \leqslant t_{i} \leqslant 1, i=1, \ldots, n, \sum_{i=1}^{n} t_{i}=1\right\}$ ). Then $0 \notin \Lambda$.

Since it is easily seen that

$$
d(0, \Lambda)=\inf \left\{\frac{\alpha \cdot \lambda}{|\alpha|}: \alpha \in g_{n}, \alpha \neq 0\right\}
$$

(where $\left.\alpha \cdot \lambda=\sum_{i=1}^{n} \alpha_{i} \lambda_{i}\right)\left(H_{2}\right)$ is equivalent to the following: there exists $c_{0}>0$ such that

$$
|\alpha \cdot \lambda| \geqslant c_{0}|\alpha| \text { for all } \alpha \in \Phi_{n} \text {. }
$$

Let us fix variables $(x, \xi)$ adapted to $S$. Given any $y(z) \in \mathrm{C}[z]]$ we write $y(x, \xi)$ for $y(z(x, \xi))$ and we expand

$$
y(x, \xi)=\sum_{\alpha \in \mathscr{I}_{n}} y_{\alpha}(\xi) x^{\alpha}
$$

so that $\left.y_{\alpha}(\xi) \in \mathrm{C}[\xi]\right]$ denotes the coefficient of $x^{\alpha}$ in this expansion, for each $\alpha \in g_{n}$.X now becomes an operator of the form

$$
\sum_{i=1}^{n} X_{i}(x, \xi) \frac{\partial}{\partial x_{i}}+\sum_{j=1}^{s} Y_{j}(x, \xi) \frac{\partial}{\partial \xi_{j}} \equiv X^{\prime}+Y
$$

where $X_{i}(0, \xi)=0, i=1, \ldots, n$, and $Y_{j}(0, \xi)=0, j=1, \ldots, s$. Expanding $X u(x, \xi)$ in the same way gives, for the coefficient of $x^{\alpha}$, the sum of two expressions of the form

$$
\left\{X^{\prime} u\right\}_{\alpha}(\xi)=\sum_{0<|\beta|<|\alpha|} \mathfrak{X}_{\alpha \beta}(\xi) u_{\beta}(\xi)
$$


and

$$
\{Y u\}_{\alpha}(\xi)=\sum_{j=1}^{s} \sum_{|\beta|<|\alpha|} \mathcal{Y}_{j \alpha \beta}(\xi) \frac{\partial u_{\beta}}{\partial \xi_{j}}(\xi)
$$

with certain coefficients $\mathscr{X}_{\alpha \beta}(\xi), \mathscr{Y}_{j \alpha \beta}(\xi)$ which depend on our choice of variables. Moreover, the coefficient of $x^{\alpha}$ in the expansion of $f(z(x, \xi)$, $u(z(x, \xi)))$ is of the form $f_{\alpha}\left(\xi, u_{0}(\xi),\left\{u_{\beta}(\xi): 0<|\beta| \leqslant|\alpha|\right\}\right)$, where, for each $\alpha \in g_{n}, f_{\alpha}$ is a $p$-tuple of polynomials in the last set of variables with coefficients which are convergent power series in the variables $\xi$ and $u_{0}(\xi)$. Thus ( 1 ) is equivalent to the infinite system of equations for the undetermined coefficients $\left.\left\{u_{\alpha}(\xi) \in \mathrm{C}[\xi]\right]^{p}: \alpha \in \mathscr{G}_{n}\right\}$ :

$$
\begin{aligned}
\sum_{0<|\beta|<|\alpha|} \mathcal{X}_{\alpha \beta}(\xi) u_{\beta}(\xi) & +\sum_{j=1}^{s} \sum_{|\beta|<|\alpha|} \mathcal{Y}_{j \alpha \beta}(\xi) \frac{\partial u_{\beta}}{\partial \xi}(\xi) \\
& =f_{\alpha}\left(\xi, u_{0}(\xi),\left\{u_{\beta}(\xi): 0<|\beta| \leqslant|\alpha|\right\}\right) .
\end{aligned}
$$

We shall specialize our choice of variables below so that $(5)_{\alpha}$ becomes easier to analyze, but our results are all independent of this choice. To state them in invariant form requires some notation. Thus, let $J$ denote the ideal of C[I]] consisting of those $y(z)$ which "vanish on $S$ " in the sense that $y(x, \xi)=0$ when $x=0$; it is easily seen that this condition is independent of our choice of variables $(x, \xi)$ adapted to $S$. Denoting by $J^{(k)}$ the ideal generated by products of $k$ elements of $J$, we observe that $y(z) \in J^{(k)}$ if and only if $y_{\alpha}(\xi)=0$ for all $\alpha$ with $|\alpha|<k$, and this condition is again independent of our choice. Consequently, for each integer $k>0$ and for each choice of variables $(x, \xi)$ we have a bijective correspondence between the residue classes $[y(z)]_{k}$ of elements $\left.y(z) \in \mathbf{C}[z]\right]$ in the quotient algebra $\left.\mathrm{C}[z]\right] / J^{(k)} \equiv$ $A_{k}$, on the one hand, and the pseudo-polynomials $\Sigma_{|\alpha|<k} y_{\alpha}(\xi) x^{\alpha}$ of degree less than $k$ in $x$, with coefficients $\left.y_{\alpha}(x) \in \mathrm{C}[\xi]\right]$, on the other; for $k=0$ both sets reduce to the one containing only the zero element. When a choice of variables $(x, \xi)$ has been made we write simply

$$
[y(z)]_{k}=\sum_{|\alpha|<k} y_{\alpha}(\xi) x^{\alpha} \text {. }
$$

We denote by $A_{k, 0}$ the image, in $A_{k}$, of $\left.\mathrm{C}[z]\right]_{0}$ under the natural projection. Define, for any $y(z) \in \mathrm{C}[z]]$,

$$
\operatorname{ord}_{s} y(z) \equiv \sup \left\{k: y(z) \in J^{(k)}\right\} \text {. }
$$

Then, clearly, for any $y(z) \in \mathrm{C}[z]]$

$$
\operatorname{ord}_{s} X y(x) \geqslant \operatorname{ord}_{s} y(z)
$$

which translates into the assertion that only the coefficients $\left\{u_{\beta}(\xi):|\beta| \leqslant\right.$ $|\alpha|\}$ appear on the left-hand side of $(5)_{\alpha}$. Given $u(z)=\left(u_{1}(z), \ldots, u_{p}(z)\right) \in$ $\mathrm{C}[\mathrm{z}]]^{p}$ we define 


$$
\operatorname{ord}_{s} u(z)=\min _{i=1, \ldots, p} \operatorname{ord}_{s} u_{i}(z),
$$

and we note that for any $\varphi(z, u) \in C[z, u]]$ and any $\left.u(z), v(z) \in[C[z]]_{0}\right]^{p}$ we have

$$
\operatorname{ord}_{s}\{\varphi(z, u(z))-\varphi(z, v(z))\} \geqslant \operatorname{ord}_{s}\{u(z)-v(z)\}
$$

(see the proof of the corollary to Lemma 2 below). Translated, this becomes the assertion that in the right-hand side of $(5)_{\alpha}$ only $\left\{u_{\beta}(\xi):|\beta| \leqslant|\alpha|\right\}$ occur. In our invariant notation, the finite system of equations $\left\{(5)_{\alpha}:|\alpha|<k\right\}$ becomes

$$
[X u(z)]_{k}=[f(z, u(z))]_{k} .
$$

Both sides of this last equation define transformations of $\left[A_{k, 0}\right]^{p}$ into itself, as we have just seen. Thus, we may define $\Phi_{k}:\left[A_{k, 0}\right]^{p} \rightarrow\left[A_{k, 0}\right]^{p}$ for each $k$ by

$$
\Phi_{k}\left([u(z)]_{k}\right)=[X u(z)]_{k}-[f(z, u(z))]_{k}
$$

and so the infinite system $\left\{(5)_{\alpha}: \alpha \in \mathscr{G}_{n}\right\}$ becomes

$$
\Phi_{k}\left([u(z)]_{k}\right)=0, \quad k=0,1,2, \ldots
$$

We need finally two last pieces of terminology: given any element $\psi$ of $A_{k}$ we say that $\psi$ is convergent if $\psi=[y(z)]_{k}$ for some $y(z) \in \mathbf{C}\{z\}$. If in the variables $(x, \xi), \psi=\Sigma_{|\alpha|<k} y_{\alpha}(\xi) x^{\alpha}$, then clearly $\psi$ is convergent if and only if each $y_{\alpha}(\xi) \in \mathbf{C}\{\xi\},|\alpha|<k$, and this is independent of which such variables we use. We say that a $p$-tuple $\psi=\left(\psi_{1}, \ldots, \psi_{p}\right) \in\left[A_{k}\right]^{p}$ is convergent if each of its components is. Given $\psi \in A_{k}$, we define

$$
\text { ord } \psi \equiv \inf \left\{\operatorname{ord} y(z):[y(z)]_{k}=\psi\right\} \text {. }
$$

It is easily seen that if $\psi=\Sigma_{|\alpha|<k} y_{\alpha}(\xi) x^{\alpha}$ in the $(x, \xi)$ variables then ord $\psi$ is precisely the order of $\Sigma_{|\alpha|<k} y_{\alpha}(\xi) x^{\alpha}$ in $\left.9[x, \xi]\right]$. As usual, if $\psi=\left(\psi_{1}, \ldots, \psi_{p}\right)$ $\in\left[A_{k}\right]^{p}$, ord $\psi=\min _{i=1, \ldots, p}$ ord $\psi_{i}$.

THEOREM 1. Suppose $\left(\mathrm{H}_{2}\right)$ holds. Given any $f(z, u) \in\left[\mathbf{C}\{z, u\}_{0}\right]^{p}$ let $F_{0}$ be the $p \times p$ matrix $\left(\partial f_{i}(0,0) / \partial u_{j}\right)_{i, j=1, \ldots, p}$ and let $k_{0}$ be the least nonnegative integer such that

$$
\lambda \cdot \alpha \text { is not an eigenvalue of } F_{0} \text { for any } \alpha \in \mathscr{G}_{n} \text { with }|\alpha|>k_{0} .
$$

(The existence of such $a k_{0}$ is a consequence of (4).) Then the map $u(z) \rightarrow \psi=$ $[u(z)]_{k_{0}}$ is a bijective correspondence between the solutions $u(z)$ of (1) in $\left[\mathrm{C}[z]_{0}\right]^{p}$ and the solutions $\psi$ in $\left[A_{k_{0}, 0}\right]^{p}$ of $\Phi_{k_{0}}(\psi)=0$. Moreover,

(i) if $u(z)$ and $u^{*}(z)$ are solutions of (1) in [C[z] $\left.]_{0}\right]^{p}$ and if $\psi=[u(z)]_{k_{0}}$ and $\psi^{*}=\left[u^{*}(z)\right]_{k_{0}}$ then

$$
\operatorname{ord}\left(\psi-\psi^{*}\right)=\operatorname{ord}\left(u(z)-u^{*}(z)\right),
$$

and

(ii) $u(z)$ is convergent if and only if $\psi$ is convergent. 
REMARK. If $k_{0}=0$, Theorem 1 asserts that (1) has a unique solution $\left.u(z) \in[C[z]]_{0}\right]^{p}$ which is, in fact, convergent.

The proof of Theorem 1 will be given in $\$ 3$ below. Note that the first assertion of Theorem 1 translates as follows: if $k_{0}$ satisfies (6), then each solution $\left\{u_{\alpha}(\xi):|\alpha|<k_{0}\right\}$ of the finite system of equations $\left\{(5)_{\alpha}:|\alpha|<k_{0}\right\}$ extends in a unique way to a solution $\left\{u_{\alpha}(\xi): \alpha \in \mathcal{G}_{n}\right\}$ of the whole infinite system $\left\{(5)_{\alpha}: \alpha \in g_{n}\right\}$. In fact, we shall see that whenever (6) holds, without assuming $\left(\mathrm{H}_{2}\right)$, for each $k \geqslant k_{0}$ the equations $\left\{(5)_{\alpha}:|\alpha|=k\right\}$ may be solved for $\left\{u_{\alpha}(\xi):|\alpha|=k\right\}$ in terms of $\left\{u_{\alpha}(\xi):|\alpha|<k\right\}$ (this is essentially Lemma 1 below). Without (6), this may fail: consider the equation

$$
z_{1} \frac{\partial u}{\partial z_{1}}-z_{2} \frac{\partial u}{\partial z_{2}}=0
$$

in which $p=1, d=2, f=0, S=\{0\}$ and $\lambda_{1}=1, \lambda_{2}=-1$, so that $\lambda \cdot \alpha=0$ $\left(=F_{0}\right)$ whenever $\alpha_{1}=\alpha_{2}$. The general solution is given by $\varphi\left(z_{1} z_{2}\right)$ where $\varphi(t) \in C[[t]]$ is an arbitrary formal power series in one variable so that no finite number of coefficients determine the whole solution.

We may read assertion (i) of Theorem 1 as follows: if $\left\{u_{\alpha}(\xi): \alpha \in \mathscr{G}_{n}\right\}$ and $\left\{u_{\alpha}^{*}(\xi): \alpha \in \mathscr{I}_{n}\right\}$ are solutions of $\left\{(5)_{\alpha}: \alpha \in \mathscr{I}_{n}\right\}$ and if $k_{0}$ satisfies (6) then

$$
\operatorname{ord}\left(\sum_{\alpha \in \mathcal{G}_{n}}\left(u_{\alpha}(\xi)-u_{\alpha}^{*}(\xi)\right) x^{\alpha}\right)=\operatorname{ord}\left(\sum_{|\alpha|<k_{0}}\left(u_{\alpha}(\xi)-u_{\alpha}^{*}(\xi)\right) x^{\alpha}\right) \text {. }
$$

Again, only (6) (and not $\left(\mathrm{H}_{2}\right)$ ) is needed here (see Lemma 2 below). The full strength of $\left(\mathrm{H}_{2}\right)$ seems to be needed for the proof of assertion (ii), given by majorant arguments (Lemmas 3-5 below). Relaxing $\left(\mathrm{H}_{2}\right)$ here leads to smalldivisor problems; we give one example to show that even if a $k_{0}$ satisfying (6) exists, assertion (ii) may fail in the absence of $\left(\mathrm{H}_{2}\right)$. Consider (with $\theta$ and $f$ to be described below) the equation

$$
z_{1} \frac{\partial u}{\partial z_{1}}-\theta z_{2} \frac{\partial u}{\partial z_{2}}=f\left(z_{1}, z_{2}\right)
$$

Here $\theta$ is taken irrational with $0<\theta<1$ so that we have $p=1, d=2$, $S=\{0\}$ and $F_{0}=0$, but $\lambda_{1}=1$ and $\lambda_{2}=-\theta$, so that $\left(\mathrm{H}_{2}\right)$ fails although (6) holds with $k_{0}=1$. We assume further about $\theta$ that it may be extremely well approximated by rationals, in the following sense: for $q=1,2, \ldots$ define

$$
\{\theta\}_{q}=\inf _{p=0,1, \ldots, q}\left|\theta-\frac{p}{q}\right| \text {. }
$$

We assume that

$$
\varliminf_{q \rightarrow \infty} \frac{1}{q} \log \{\theta\}_{q}=-\infty .
$$

(Take, e.g., $\theta=\sum_{j=1}^{\infty} 2^{-n_{j}}$ where $\left\{n_{j}\right\}_{j=1}^{\infty}$ is any increasing sequence of positive integers such that $n_{j+1} / 2^{n_{j}} \rightarrow \infty$ as $j \rightarrow \infty$.) To construct $f\left(z_{1}, z_{2}\right)$ take an 
increasing sequence $\left\{q_{j}\right\}_{j=1}^{\infty}$ of positive integers such that $q_{j}^{-1} \log \{\theta\}_{q_{j}} \rightarrow-\infty$ (in the example $\theta=\sum_{j=1}^{\infty} 2^{-n_{j}}, q_{j}=2^{n_{j}}$ will do) and, for $j=1,2, \ldots$, let $p_{j}$ be the integer $p$ between 0 and $q_{j}$ at which $\left|\theta-p / q_{j}\right|$ attains its minimum value $\{\theta\}_{q_{j}}$. Now define $f\left(z_{1}, z_{2}\right)=\sum_{j=1}^{\infty} z_{1}^{p_{i}} z_{2}^{q_{j}}$ which is certainly convergent. The unique formal power series solution $u\left(z_{1}, z_{2}\right)$ of $(7)$ is given by

$$
u\left(z_{1}, z_{2}\right)=\sum_{j=1}^{\infty} \frac{z_{1}^{p_{j}} z_{2}^{q_{j}}}{p_{j}-\theta q_{j}}
$$

if we take $z_{1} \neq 0$ and $z_{2} \neq 0$, and write $\rho \equiv \min \left(\left|z_{1}\right|,\left|z_{2}\right|\right)>0$, then

$$
\left|\frac{z_{1}^{p_{j}} z_{2}^{q_{j}}}{p_{j}-\theta q_{j}}\right| \geqslant \frac{\rho^{p_{j}+q_{j}}}{q_{j}\{\theta\}_{q_{j}}} \equiv \eta_{j},
$$

and

$$
\log \eta_{j}=\left(p_{j}+q_{j}\right) \log \rho-\log q_{j}-\log \{\theta\}_{q_{j}} \rightarrow+\infty \quad \text { as } j \rightarrow \infty,
$$

so that $u\left(z_{1}, z_{2}\right)$ is not convergent.

THEOREM 2. Suppose $\left(\mathrm{H}_{2}\right)$ holds. Then there exist variables $(x, \xi)$ adapted to $S$ in which $X$ becomes $X^{\prime}=\sum_{i=1}^{n} X_{i}(x, \xi) \partial / \partial x_{i}$ (so that $Y=0$, i.e., the variables $\xi_{1}, \ldots, \xi_{s}$ appear in $X$ only as parameters).

Proof. Let $\left(x^{\prime}, \xi^{\prime}\right)$ be any variables adapted to $S$. We shall show the existence of solutions $\xi_{1}\left(x^{\prime}, \xi\right), \ldots, \xi_{s}\left(x^{\prime}, \xi^{\prime}\right) \in \mathbf{C}\left\{x^{\prime}, \xi^{\prime}\right\}$ of the equation

$$
X \xi=0
$$

such that

$$
\xi_{j}\left(0, \xi^{\prime}\right)=\xi_{j}^{\prime} \text { for } j=1, \ldots, s .
$$

The variables $(x, \xi)$ defined by

$$
\begin{aligned}
& x_{i}=x_{i}^{\prime}, \quad i=1, \ldots, n, \\
& \xi_{j}=\xi_{j}\left(x^{\prime}, \xi^{\prime}\right), \quad j=1, \ldots, s,
\end{aligned}
$$

will then clearly have the desired properties. But (8) is a special case of (1), with $p=1$ and $f=0$; (6) holds in this case with $k_{0}=1$. We define $\psi_{j} \in A_{1,0}$ by setting $\psi_{j}=\xi_{j}^{\prime}$ in the variables $\left(x^{\prime}, \xi\right)$; we have $\Phi_{1}\left(\psi_{j}\right)=0$ since $X \xi_{j}^{\prime}=$ $Y_{j}\left(x^{\prime}, \xi^{\prime}\right) \in J$ for $j=1, \ldots, s$. We may therefore apply Theorem 1 to conclude our proof.

The following examples are meant to put Theorem 2 in perspective. In all three we have $d=3, S=\left\{z \in C^{3}: z_{1}=z_{2}=0\right\}$ so that the equations $x_{1}=$ $z_{1}, x_{2}=z_{2}, \xi_{1}=z_{3}$ define variables $\left(x_{1}, x_{2}, \xi_{1}\right)$ adapted to $S$.

$$
X=z_{1} \frac{\partial}{\partial z_{1}}-z_{2} \frac{\partial}{\partial z_{2}}+z_{1} z_{2} \frac{\partial}{\partial z_{3}} \text {. }
$$

Here, $\lambda_{1}=1$ and $\lambda_{2}=-1$, so $\left(\mathrm{H}_{2}\right)$ fails, as does (6), for any $k_{0}$. Moreover 
there is not even any formal power series solution $\xi(z)$ of $(8)$ with $\partial \xi(0) / \partial z_{3} \neq$ 0 , as is easily seen by calculating the coefficient of $z_{1} z_{2}$ in the expansion of $X \xi(z)$; thus Theorem 2 fails here.

$$
X=z_{1} \frac{\partial}{\partial z_{1}}-z_{2} \frac{\partial}{\partial z_{2}}+z_{1} z_{3} \frac{\partial}{\partial z_{3}} .
$$

Here again we have $\lambda_{1}=1$ and $\lambda_{2}=-1$, but now a solution $\xi(z)=z_{3} e^{-z_{1}}$ of (8) with $\partial \xi(0) / \partial z_{3}=1$ is at hand. Thus Theorem 2 is not the last word on the subject.

$$
X=z_{1} \frac{\partial}{\partial z_{1}}-\theta z_{2} \frac{\partial}{\partial z_{2}}-f\left(z_{1}, z_{2}\right) z_{3} \frac{\partial}{\partial z_{3}}
$$

where $\theta$ and $f\left(z_{1}, z_{2}\right)$ are as in the example (7) above. Suppose $\xi(z)$ is a formal power series solution of (8) with $\partial \xi(0) / \partial z_{3} \neq 0$; we may assume then that $\partial \xi(0) / \partial z_{3}=1$. If we expand

$$
\xi\left(z_{1}, z_{2}, z_{3}\right)=\sum_{k=0}^{\infty} \xi_{k}\left(z_{1}, z_{2}\right) z_{3}^{k}
$$

(8) becomes

$$
\left(z_{1} \frac{\partial}{\partial z_{1}}-\theta z_{2} \frac{\partial}{\partial z_{2}}\right) \xi_{k}\left(z_{1}, z_{2}\right)=k f\left(z_{1}, z_{2}\right) \xi_{k}\left(z_{1}, z_{2}\right), \quad k=0,1,2, \ldots
$$

In particular, since $\xi_{1}(0,0)=1, u\left(z_{1}, z_{2}\right) \equiv \log \xi_{1}\left(z_{1}, z_{2}\right)$ is a formal power series solution of (7), and so, as we have seen, is not convergent. Thus, in this case, a change of variables as described in Theorem 2 may be effected with formal power series, but not with convergent ones.

THEOREM 3. Suppose $\left(\mathrm{H}_{2}\right)$ holds. Given any solution $\left.u(z) \in[\mathrm{C}[z]]_{0}\right]^{p}$ of (1) and any integer $\nu \geqslant 0$ there exists $\tilde{u}(z) \in\left[C\{z\}_{0}\right\}^{p}$ which also satisfies (1) and is such that

$$
\operatorname{ord}(u(z)-\tilde{u}(z)) \geq \nu .
$$

Proof. Let $k_{0}$ be as in Theorem 1; by the remark following Theorem 1 we may assume that $k_{0} \geqslant 1$. It suffices to find a convergent element $\psi$ of $\left[A_{k_{0}, 0}\right]^{p}$ satisfying $\Phi_{k_{0}}(\psi)=0$ and such that

$$
\operatorname{ord}\left([u(z)]_{k_{0}}-\psi\right)>\nu \text {. }
$$

To that end, choose variables $(x, \xi)$ as in Theorem 2. Writing $\psi=$ $\Sigma_{|\alpha|<k_{0}} \psi_{\alpha}(\xi) x^{\alpha}$ in these variables and translating the equation $\Phi_{k_{0}}(\psi)=0$ (using $\left\{(5)_{\alpha}:|\alpha|<k_{0}\right\}$, with all the $\mathscr{Y}_{j \alpha \beta}=0$ ) we get

$$
\sum_{0<|\beta|<|\alpha|} \mathscr{X}_{\alpha \beta}(\xi) \psi_{\beta}(\xi)=f_{\alpha}\left(\xi, \psi_{0}(\xi),\left\{\psi_{\beta}(\xi): 0<|\beta|<|\alpha|\right\}\right)
$$


for all $\alpha$ with $|\alpha|<k_{0}$ for the equations to be satisfied by the $\left\{\psi_{\alpha}(\xi)\right.$ :

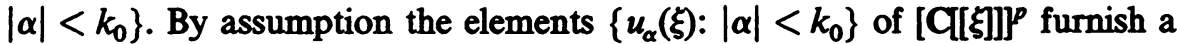
solution of (9); moreover, $u_{0}(\xi) \in\left[\mathcal{C}[\xi]_{0}\right]^{p}$ and the $f_{\alpha}$ are polynomials in the variables $\left\{\psi_{\beta}: 0<|\beta| \leqslant|\alpha|\right\}$ so a trivial modification of Artin's theorem [1] gives us the existence of $\left\{\psi_{\alpha}(\xi) \in[C\{\xi\}]^{p}:|\alpha|<k_{0}\right\}$, with $\psi_{0}(\xi) \in\left[C\{\xi\}_{0}\right]^{p}$, satisfying (9) and such that

$$
\operatorname{ord}\left(u_{\alpha}(\xi)-\psi_{\alpha}(\xi)\right) \geqslant \nu \text { for all } \alpha \text { with }|\alpha| \leqslant k_{0} .
$$

Then

$$
\begin{aligned}
\operatorname{ord}\left([u(z)]_{k_{0}}-\psi\right) & =\operatorname{ord}\left(\sum_{|\alpha|<k_{0}}\left\{u_{\alpha}(\xi)-\psi_{\alpha}(\xi)\right\} x^{\alpha}\right) \\
& \geqslant \min _{|\alpha|<k_{0}}\left\{|\alpha|+\operatorname{ord}\left(u_{\alpha}(\xi)-\psi_{\alpha}(\xi)\right)\right\}>\nu .
\end{aligned}
$$

ReMARK. Counterexamples to Theorem 3 (failing $\left(\mathrm{H}_{2}\right)$ ) are provided by the two examples at the end of $\S 1$, the example given in $(7)$ above, and the example (iii) given just before Theorem 3 .

3. Proof of Theorem 1. Given $\psi \in\left[A_{k_{0} 0}\right]^{p}$ satisfying $\Phi_{k_{0}}(\psi)=0$, we seek a (unique) $\left.u(z) \in[\mathrm{C}[z]]_{0}\right]^{p}$ satisfying (1) and $[u(z)]_{k_{0}}=\psi$. Simple arguments which we give at the end of this section enable us to reduce the general case to the apparently special one where $k_{0}>1$ and $\psi=0$, which we treat in the following lemmas. Note that $\Phi_{k_{0}}(0)=0$ means precisely that ord $f(z, 0)>k_{0}$ while $[u(z)]_{k_{0}}=0$ means that $\operatorname{ord}_{s} u(z)>k_{0}$.

LEMMA 1. Given an integer $k>1$ and $f(z, u) \in\left[\mathrm{C}[z, u]_{0}\right]^{p}$ such that

(a) ord $f(z, 0)>k$, and

(b) $\lambda \cdot \alpha$ is not an eigenvalue of $F_{0} \equiv\left(\partial f_{i}(0,0) / \partial u_{j}\right)_{i j-1, \ldots, p}$ for any $\alpha \in G_{n}$ with $|\alpha|>k$. Then there exists a unique solution $\left.u(z) \in[\mathrm{C}[z]]_{0}\right]^{p}$ of (1) satisfying ord $u(z)>k$.

Proof. For the moment, choose any variables $(x, \xi)$ adapted to $S$. We rewrite (5) $)_{\alpha}$ so as to isolate the terms $\left\{u_{\beta}(\xi):|\beta|=|\alpha|\right\}$ of highest order. To this end, let $F(\xi)$ denote the matrix $\left(\partial f_{i}(z(0, \xi), 0) / \partial u_{j}\right)_{i, j-1, \ldots, p}$ and define $\left.f^{(1)}(x, \xi, u) \in[\mathrm{C}[x, \xi, u]]_{0}\right]^{p}$ by means of

$$
f(z(x, \xi), u)=F(\xi) u+f^{(1)}(x, \xi, u)
$$

with the obvious vector notation; clearly then $F(0)=F_{0}$ and

Rewriting (1) in the form

$$
\frac{\partial f_{i}^{(1)}}{\partial u_{j}}(0, \xi, 0)=0 \text { for } i, j=1, \ldots, p .
$$

$$
\begin{aligned}
& \left\{\sum_{i j=1}^{n} \frac{\partial X_{i}}{\partial x_{j}}(0, \xi) x_{j} \frac{\partial}{\partial x_{i}}-F(\xi)\right\} u(x, \xi) \\
& \quad=f^{(1)}(x, \xi, u(x, \xi))-\left(X^{(1)}+Y\right) u(x, \xi)
\end{aligned}
$$


where we have written $X^{(1)}$ for $\sum_{i=1}^{n} X_{i}^{(1)}(x, \xi) \partial / \partial x_{i}$ with

$$
X_{i}^{(1)}(x, \xi)=X_{i}(x, \xi)-\sum_{j=1}^{n} \frac{\partial X_{i}}{\partial x_{j}}(0, \xi) x_{j}, \quad i=1, \ldots, n,
$$

we see, comparing with (5) $)_{\alpha}$ that the coefficient of $x^{\alpha}$ on the left-hand side of (11) is precisely

$$
\sum_{|\beta|=|\alpha|} \mathcal{X}_{\alpha \beta}(\xi) u_{\beta}(\xi)-F(\xi) u_{\alpha}(\xi)=\sum_{|\beta|=|\alpha|}\left\{\mathcal{X}_{\alpha \beta}(\xi) I-F(\xi) \delta_{\alpha \beta}\right\} u_{\beta}(\xi)
$$

where $I$ denotes the identity element in $M_{p}(C)$, the ring of $p \times p$ matrices with entries in $\mathbf{C}$, while the coefficient of $x^{\alpha}$ on the right-hand side involves only $\left\{u_{\beta}(\xi):|\beta|<|\alpha|\right\}$ and their first derivatives in $\xi_{1}, \ldots, \xi_{s}$. We shall show that for each $l \geqslant k$, the finite system $\left\{(5)_{\alpha}:|\alpha|=l\right\}$ may be solved for $\left\{u_{\alpha}(\xi)\right.$ : $|\alpha|=l\}$, at least if we choose our variables $(x, \xi)$ appropriately. Clearly, for our purpose it would suffice to show that there exists even one such choice of variables adapted to $(x, \xi)$ for which this holds; it is, in fact, not hard to see that it must then also be so for any such choice of variables. Since for any variables $(x, \xi)$ adapted to $S$,

$$
\frac{\partial Y_{j}}{\partial \xi_{k}}(0,0)=0, \quad j, k=1, \ldots, s,
$$

it follows that the numbers $\lambda_{1}, \ldots, \lambda_{n}$ are the eigenvalues of the matrix

$$
\left(\frac{\partial X_{i}}{\partial x_{j}}(0,0)\right)_{i, j=1, \ldots, n} \equiv A \text {. }
$$

We may then assume that $(x, \xi)$ has been chosen (applying an invertible linear transformation $x^{\prime}=T x$, if necessary) so that $A$ is in Jordan canonical form, i.e., that

$$
A=\left\|\begin{array}{ccccc}
\lambda_{1} & \mu_{1} & 0 & \cdots & 0 \\
0 & \lambda_{2} & \mu_{2} & \cdots & 0 \\
\vdots & \vdots & \vdots & & \vdots \\
0 & \cdot & \cdot & \cdots & \lambda_{n}
\end{array}\right\|
$$

where $0<\mu_{i} \leqslant 1$ for $i=1, \ldots, n-1$. To complete our proof, we order $g_{n, l} \equiv\left\{\alpha \in g_{n}:|\alpha|=l\right\}$ lexicographically, so that $\alpha>\beta$ means that there is an $i, 1<i<n$, such that $\alpha_{j}=\beta_{j}$ for all $j<i$ but $\alpha_{i}>\beta_{i}$. With this ordering, the array

$$
\left\{\mathscr{X}_{\alpha \beta}(\xi) I-F(\xi) \delta_{\alpha \beta}\right\}_{\alpha, \beta \in \Phi_{n, d}}
$$

of elements of $M_{p}\left(\mathrm{C}[\xi] \mathrm{D}\right.$ becomes an element $\Gamma^{(l)}(\xi)$ of $\left.M_{p N_{l}}(\mathrm{C}[\xi]]\right)$ where $N_{l}$ denotes the cardinality of $g_{n, l}$. In fact, we shall show that $\Gamma^{(n)}(\xi)$ is invertible 
in $M_{p N_{t}}\left(\mathrm{C}[\xi] \mathrm{D}\right.$ for $l \geqslant k$, which amounts to our assertion that $\left\{(5)_{\alpha}:|\alpha|=l\right\}$ may be solved for $\left\{u_{\alpha}(\xi):|\alpha|=l\right\}$. Clearly, it suffices to show that $\Gamma^{(I)}(0)$ is invertible in $M_{p N_{t}}(C)$. Since $\Sigma_{\beta \in \mathcal{I}_{n, 1}} \mathfrak{X}_{\alpha \beta}(0) u_{\beta}(\xi)$ is the coefficient of $x^{\alpha}$ in the expansion of

$$
\sum_{i, j=1}^{n} \frac{\partial X_{i}}{\partial x_{j}}(0,0) x_{j} \frac{\partial u}{\partial x_{i}}(x, \xi)
$$

it follows that $\mathscr{X}_{\alpha \alpha}(0)=\lambda \cdot \alpha$ for all $\alpha \in \mathscr{I}_{n, l}$ and $\mathscr{X}_{\alpha \beta}(0)=0$ if $\alpha>\beta$, $\alpha, \beta \in \mathscr{I}_{n, l}$. Thus

$$
\operatorname{det} \Gamma^{(l)}(0)=\prod_{\alpha \in \Phi_{n, l}} \operatorname{det}\left((\lambda \cdot \alpha) I-F_{0}\right) \neq 0
$$

if $l>k$, by (b), and our proof is complete.

LEMMA 2. Given an integer $k \geqslant 1$ and a $p \times p$ matrix $C(z)$ of formal power series such that $\lambda \cdot \alpha$ is not an eigenvalue of $C(0)$ for any $\alpha \in G_{n}$ with $|\alpha|>k$, suppose $\left.u(z) \in[C[z]]_{0}\right]^{p}$ satisfies $X u(z)=C(z) u(z)$. Then

$$
\text { ord } u(z)=\operatorname{ord}\left([u(z)]_{k}\right) \text {. }
$$

Proof. Choose variables $(x, \xi)$ as in the proof of Lemma 1. Define, for $l=0,1,2, \ldots$,

$$
U_{l}(x, \xi)=\sum_{\alpha \in g_{n, l}} u_{\alpha}(\xi) x^{\alpha} .
$$

Since $u(x, \xi)=\sum_{l=0}^{\infty} U_{l}(x, \xi)$ it suffices to show that

$$
\operatorname{ord} U_{l}(x, \xi)>\operatorname{ord}\left(\sum_{i=0}^{k-1} U_{i}(x, \xi)\right) \text { for } l>k \text {. }
$$

This, in turn, is an immediate consequence of the assertion that

$$
\text { ord } U_{l}(x, \xi) \geqslant \operatorname{ord}\left(\sum_{i=0}^{l-1} U_{i}(x, \xi)\right) \text { for } l \geqslant k
$$

which is, in fact, what we shall prove. Now we set

$$
F(\xi)=C(z(0, \xi))
$$

and

$$
C^{\prime}(x, \xi)=C(z(x, \xi))-F(\xi)
$$

so that, as in the proof of Lemma 1, the equation $X u(z)=C(z) u(z)$ may be rewritten as

$$
\left\{\sum_{i, j=1}^{n} \frac{\partial X_{i}}{\partial x_{j}}(0, \xi) x_{j} \frac{\partial}{\partial x_{i}}-F(\xi)\right\} u(x, \xi)=\left(C^{\prime}(x, \xi)-X^{(1)}-Y\right) u(x, \xi) .
$$

We expand both sides as in the proof of Lemma 1 . If we denote by $v_{\alpha}(\xi)$ the coefficient of $x^{\alpha}$ on either side, then we have 


$$
v_{\alpha}(\xi)=\sum_{\beta \in \Phi_{n, l}}\left\{\mathscr{X}_{\alpha \beta}(\xi) I-F(\xi) \delta_{\alpha \beta}\right\} u_{\beta}(\xi)
$$

If $|\alpha|=l, v_{\alpha}(\xi)$ is clearly also the coefficient of $x^{\alpha}$ in the expansion of

$$
\left(C^{\prime}(x, \xi)-X^{(1)}-Y\right) \sum_{i=0}^{l-1} U_{i}(x, \xi)
$$

and so

$$
\text { ord } \begin{aligned}
v_{\alpha}(\xi)+l & \geqslant \operatorname{ord}\left\{\left(C^{\prime}(x, \xi)-X^{(1)}-Y\right)\left(\sum_{i=0}^{l-1} U_{i}(x, \xi)\right)\right\} \\
& \geqslant \operatorname{ord} \sum_{i=0}^{l-1} U_{i}(x, \xi) .
\end{aligned}
$$

On the other hand, it follows from the invertibility of $\Gamma^{(t)}(\xi)$ that, for $|\alpha|=l \geqslant k$,

$$
\operatorname{ord} u_{\alpha}(\xi) \geqslant \min _{|\beta|=l} \operatorname{ord} v_{\beta}(\xi) \geqslant \operatorname{ord}\left(\sum_{i=0}^{l-1} U_{i}(x, \xi)\right)-l .
$$

As

$$
\text { ord } U_{l}(x, \xi)=\min _{|\alpha|=l} \text { ord } u_{\alpha}(\xi)+l,
$$

our proof is complete.

COROLlary. Given an integer $k \geqslant 1$ and $\left.f(z, u) \in[\mathrm{C}[z, u]]_{0}\right]^{p}$ satisfying condition (b) of Lemma 1, suppose that $u(z)$ and $v(z)$, both in $\left.[\mathrm{C}[z]]_{0}\right]^{p}$, are solutions of (1). Then

$$
\operatorname{ord}\{u(z)-v(z)\}=\operatorname{ord}\left\{[u(z)-v(z)]_{k}\right\} .
$$

Proof. We write $f(z, u)-f(z, v)=H(z, u, v)(u-v)$ where $H(z, u, v)$ is a $p \times p$ matrix of elements of $\mathcal{C}[z, u, v]]$ with

$$
H(0,0,0)=F_{0}=\left(\frac{\partial f_{i}}{\partial u_{j}}(0,0)\right)_{i, j=1, \ldots, p} .
$$

If we define $C(z)=H(z, u(z), v(z))$ and $w(z)=u(z)-v(z)$, then we have $X w(z)=C(z) w(z)$, and our assertion follows at once from Lemma 2 .

REMARK. Both Lemma 2 and its corollary remain true when the orders in question are infinite (e.g., for the corollary, $u(z)=v(z)$ if and only if $\left.[u(z)]_{k}=[v(z)]_{k}\right)$. In fact, the reasoning is exactly the same in this case.

DEFINITION. If

$$
y(z)=\sum_{\alpha \in \Phi_{d}} y_{\alpha} z^{\alpha} \text { and } y^{*}(z)=\sum_{\alpha \in \Phi_{d}} y_{\alpha}^{*} z^{\alpha}
$$

are in C[Iz]], we say that $y(z) \prec y^{*}(z)$ if $\left|y_{\alpha}\right|<y_{\alpha}^{*}$ for all $\alpha \in \mathscr{I}_{d}$. For 
$u(z)=\left(u_{1}(z), \ldots, u_{p}(z)\right)$ and $u^{*}(z)=\left(u_{1}^{*}(z), \ldots, u_{p}^{*}(z)\right)$ in $\left.[\mathrm{C}[z]]\right]^{p}, u(z) \prec$ $u^{*}(z)$ means that $u_{i}(z)<u_{i}^{*}(z)$ for all $i=1,2, \ldots, p$. We write $\left.\mathrm{C}[z]\right]_{+}$for the subset of $\mathrm{C}[z]]$ consisting of those power series all of whose coefficients are nonnegative; similarly for $\mathbf{C}\{z\}_{+}$. Finally, we write $|y|(z)$ for $\Sigma_{\alpha \in \mathcal{I}_{n}}\left|y_{\alpha}\right| z^{\alpha}$, the least majorant of $y(z)$, and

$$
|u|(z)=\left(\left|u_{1}\right|(z),\left|u_{2}\right|(z), \ldots,\left|u_{p}\right|(z)\right) .
$$

LeMMA 3. Given $A(z)$, a $p \times p$ matrix of elements of $\mathrm{C}[z]]_{+}$, let $\rho$ be the largest eigenvalue, in absolute value, of $A(0)$. (By a theorem of Frobenius and Perron, $\rho>0$; see, for example, [3, Chapter XIII, Theorem 3].) Let $\rho<1$. Then $(I-A(z))^{-1}$ exists, in the ring of $p \times p$ matrices with entries in $\left.\mathrm{Q}[z]\right]$. In fact, $(I-A(z))^{-1}$ has entries in $\left.\mathrm{q}[z]\right]_{+}$, and is convergent whenever $A(z)$ is. Moreover, if $u(z)$ and $v(z)$ are elements of $\left.[G[z]]_{+}\right]^{p}$ such that

$$
u(z)<A(z) u(z)+v(z)
$$

then

$$
u(z)<(I-A(z))^{-1} v(z) .
$$

Proof. We write $A(z)=A(0)+A^{\prime}(z)$. Our proof proceeds by cases.

(i) Suppose $A^{\prime}(z)=0$. We know that $\lim _{m \rightarrow \infty}\left\|A(0)^{m}\right\|^{1 / m}=\rho<1$, whenever $\|\cdot\|$ is any norm which makes the ring of $p \times p$ matrices with complex entries into a Banach algebra (see [2, p. 864]). Therefore, the series $\sum_{l=0}^{\infty} A(0)^{l}$ converges to $(I-A(0))^{-1}$; in particular, $(I-A(0))^{-1}$ has nonnegative entries, and $\lim _{m \rightarrow \infty} A(0)^{m}=0$. Furthermore, we have

$$
\begin{aligned}
u(z) & <A(0) u(z)+v(z) \prec A(0)^{2} u(z)+(A(0)+I) v(z) \\
& <A(0)^{3} u(z)+\left(A(0)^{2}+A(0)+I\right) v(z) \\
& \vdots \\
& <A(0)^{m+1} u(z)+\left\{\sum_{l=0}^{m} A(0)^{l}\right\} v(z),
\end{aligned}
$$

for $m=0,1,2, \ldots$ Letting $m \rightarrow \infty$ gives

(ii) Suppose $A(0)=0$. We have

$$
u(z)<(I-A(0))^{-1} v(z) \text {. }
$$

$$
\sum_{l=0}^{\infty} A^{\prime}(z)^{l}=\left(I-A^{\prime}(z)\right)^{-1},
$$

the sum converging in the usual formal power series sense. As is well known $\left(I-A^{\prime}(z)\right)^{-1}$ is convergent whenever $A^{\prime}(z)$ is. As in (i), we have

$$
u(z) \prec A^{\prime}(z)^{m+1} u(z)+\left\{\sum_{l=0}^{m} A^{\prime}(z)^{l}\right\} v(z)
$$


for $m=0,1,2, \ldots$ Letting $m \rightarrow \infty$ now gives

$$
u(z)<\left(I-A^{\prime}(z)\right)^{-1} v(z) \text {. }
$$

(iii) For the general case, we set $B(z)=(I-A(0))^{-1} A^{\prime}(z)$. Then $B(0)=0$, and an easy calculation shows that

$$
(I-A(z))^{-1}=(I-B(z))^{-1}(I-A(0))^{-1},
$$

the right-hand side making sense because of (i) and (ii); appealing to these two special cases gives further that

$$
u(z)<(I-A(0))^{-1}\left(A^{\prime}(z) u(z)+v(z)\right)
$$

and therefore

$$
u(z)<(I-B(z))^{-1}(I-A(0))^{-1} v(z)
$$

which completes our proof.

Throughout the remainder of our work $t$ is used to denote a single variable.

LEMMA 4. Suppose $\left.v(t, \xi) \in[\mathrm{C}[t, \xi]]_{+}\right]^{p}$ satisfies $v(0, \xi)=0$ and

$$
t \frac{\partial v}{\partial t}(t, \xi) \prec h\left(t, \xi, v(t, \xi),\left\{\frac{\partial v_{i}}{\partial \xi_{j}}(t, \xi): i=1, \ldots, p ; j=1, \ldots, s\right\}\right)
$$

where $h(t, \xi, v, q) \in\left[\mathbf{C}\{t, \xi, v, q\}_{+}\right]^{p}\left(v=\left(v_{1}, \ldots, v_{p}\right)\right.$ and $q=\left\{q_{i j}: i=\right.$ $1, \ldots, p ; j=1, \ldots, s\})$ and where we suppose that

$$
\frac{\partial h_{i}}{\partial v_{j}}(0,0,0,0)=0 \text { for } i, j=1, \ldots, p
$$

and

$$
\frac{\partial h_{i}}{\partial q_{j k}}(0, \xi, 0,0)=0 \text { for } i, j=1, \ldots, p ; k=1, \ldots, s .
$$

Then, in fact, $v(t, \xi) \in\left[\mathbf{C}\{t, \xi\}_{+}\right]^{p}$.

Proof. We may assume, without loss of generality, that $h(0, \xi, 0,0)=0$ since, in any case, $h$ may be modified to bring this about without any effect on our hypotheses. We may also replace (14) by the stronger hypothesis

$$
\frac{\partial h_{i}}{\partial v_{j}}(0, \xi, 0,0)=0 \text { for } i, j=1, \ldots, p
$$

as the following argument shows: Write $h(t, \xi, v, q)=H(\xi) v+h^{\prime}(t, \xi, v, q)$ where $H(\xi)$ is the $p \times p$ matrix $\left(\partial h_{i}(0, \xi, 0,0) / \partial v_{j}\right)_{i, j=1, \ldots, p}$ with entries in $\mathbf{C}\{\xi\}_{+}$and $h^{\prime}(t, \xi, v, q)$ is in $\left[\mathbf{C}\{t, \xi, v, q\}_{+}\right]^{p}$ and also satisfies (14) and (15). Since $\left.v(t, \xi) \in[C[t, \xi]]_{+}\right]^{p}$ and $v(0, \xi)=0$, we have

$$
v(t, \xi)<t \frac{\partial v}{\partial t}(t, \xi)
$$


so (13) leads to

$$
t \frac{\partial v}{\partial t}(t, \xi) \prec H(\xi)\left(t \frac{\partial v}{\partial t}(t, \xi)\right)+h^{\prime}\left(t, \xi, v(t, \xi),\left\{\frac{\partial v_{i}}{\partial \xi_{j}}(t, \xi)\right\}\right) .
$$

Because we are assuming that (14) holds for $h$, we have $H(0)=0$, so applying Lemma 3 gives us

$$
t \frac{\partial v}{\partial t}(t, \xi)<\bar{h}\left(t, \xi, v(t, \xi),\left\{\frac{\partial v_{i}}{\partial \xi_{j}}(t, \xi)\right\}\right)
$$

where $\bar{h}(t, \xi, v, q)=(I-H(\xi))^{-1} h^{\prime}(t, \xi, v, q)$ which is in $\left[\mathbf{C}\{t, \xi, v, q\}_{+}\right]^{p}$ and satisfies (14) and (15). Thus, we replace (14) by (14') and revert to our original notation. We now write

$$
h(t, \xi, v, q)=t h_{1}(\xi)+\tilde{h}(t, \xi, v, q)
$$

with

$$
h_{1}(\xi)=\frac{\partial h}{\partial t}(0, \xi, 0,0) \in\left[C\{\xi\}_{+}\right]^{p}
$$

and $\tilde{h}(t, \xi, v, q)$ expressible as a finite sum

$$
\sum h_{k \alpha \beta}(t, \xi, v, q) t^{k} v^{\alpha} q^{\beta}
$$

where the sum is over these $k \in \mathscr{G}_{1}(=N), \alpha \in \mathscr{I}_{p}$ and $\beta \in \mathscr{G}_{p s}$ for which $k+|\alpha|+|\beta|=2$, and each $h_{k \alpha \beta}(t, \xi, v, q)$ is in $\left[C\{t, \xi, v, q\}_{+}\right\}^{p}$. Define $\left.w(t, \xi) \in[C[t, \xi]]_{+}\right]^{p}$ by $v(t, \xi)=t w(t, \xi)$; then (13) gives

$$
\begin{aligned}
& t^{2} \frac{\partial w}{\partial t}(t, \xi)+t w(t, \xi) \\
& \quad<t h_{1}(\xi)+t^{2} \sum h_{k \alpha \beta}\left(t, \xi, t w(t, \xi),\left\{t \frac{\partial w_{i}}{\partial \xi_{j}}(t, \xi)\right\}\right)(w(t, \xi))^{\alpha}\left(\left\{\frac{\partial w_{i}}{\partial \xi_{j}}(t, \xi)\right\}\right)^{\beta} .
\end{aligned}
$$

Therefore $w(0, \xi) \prec h_{1}(\xi)$ and

$t^{2} \frac{\partial w}{\partial v}(t, \xi)<t^{2} \sum h_{k \alpha \beta}\left(t, \xi, t w(t, \xi),\left\{t \frac{\partial w_{i}}{\partial \xi_{j}}(t, \xi)\right\}\right)(w(t, \xi))^{\alpha}\left(\left\{\frac{\partial w_{i}}{\partial \xi_{j}}(t, \xi)\right\}\right)^{\beta}$

so

$$
\frac{\partial w}{\partial t}(t, \xi) \prec \sum h_{k \alpha \beta}\left(t, \xi, t w(t, \xi),\left\{t \frac{\partial w_{i}}{\partial \xi_{j}}(t, \xi)\right\}\right)(w(t, \xi))^{\alpha}\left(\left\{\frac{\partial w_{i}}{\partial \xi_{j}}(t, \xi)\right\}\right)^{\beta} .
$$

By the Cauchy-Kowalewski theorem (see [4, Chapter 1], for example) the equation

$$
\frac{\partial w^{*}}{\partial t}(t, \xi)=\sum h_{k \alpha \beta}\left(t, \xi, t w^{*}(t, \xi),\left\{t \frac{\partial w_{i}^{*}}{\partial \xi_{j}}(t, \xi)\right\}\right)\left(w^{*}(t, \xi)\right)^{\alpha}\left(\left\{\frac{\partial w_{i}^{*}}{\partial \xi_{j}}(t, \xi)\right\}\right)^{\beta}
$$


has a unique solution $w^{*}(t, \xi) \in[C[[t, \xi]]]^{p}$ satisfying $w^{*}(0, \xi)=h_{1}(\xi)$; most important for us is that $w^{*}(t, \xi)$ is convergent. The convergence of $w(t, \xi)$, and hence of $v(t, \xi)$, follows from the fact that $w(t, \xi) \prec w^{*}(t, \xi)$, which is easily seen: write

$$
w(t, \xi)=\sum_{l=0}^{\infty} w_{l}(\xi) t^{l} \text { and } w^{*}(t, \xi)=\sum_{l=0}^{\infty} w_{l}^{*}(\xi) t^{l}
$$

and then observe, as in the proof of the Cauchy-Kowalewski theorem, that the assertion $w_{l}(\xi) \prec w_{l}^{*}(\xi)$ for all $l=0,1,2, \ldots$ follows by induction on $l$. This completes our proof.

LEMMA 5. We suppose that $\left(\mathrm{H}_{2}\right)$ holds and that an integer $k>1$ and $f(z, u) \in\left[\mathbf{C}\{z, u\}_{0}\right\}^{p}$ are given, satisfying conditions (a) and (b) of Lemma 1 . Then if $\left.u(z) \in[\mathrm{C}[z]]_{0}\right]^{p}$ is a solution of (1) such that $\operatorname{ord}_{s} u(z)>k$ (a unique such $u(z)$ exists, by Lemma 1) $u(z)$ is, in fact, convergent.

Proof. For $\alpha \in G_{n}$, with $|\alpha| \geqslant k$, let $M^{(\alpha)}$ denote the $p \times p$ matrix

$$
\left(I-\frac{1}{\lambda \cdot \alpha} F_{0}\right)^{-1}
$$

Then $M^{(\alpha)} \rightarrow I$ as $|\alpha| \rightarrow \infty$ by (4), so for each $i, j=1, \ldots, p$,

$$
M_{i j} \equiv \sup \left\{\left|M_{i j}^{(\alpha)}\right|: \alpha \in G_{n},|\alpha|>k\right\}<\infty \text {. }
$$

(4) implies that, for $|\alpha| \geqslant k$, each entry of $\left((\lambda \cdot \alpha) I-F_{0}\right)^{-1}$ is dominated, in absolute value, by the corresponding entry of the matrix $\left(1 / c_{0}|\alpha|\right) M$. Choose $\varepsilon>0$ so small that

$$
\text { the largest eigenvalue of }\left(2(n-1) \varepsilon / c_{0}\right) M \text { is less than } 1 \text {. }
$$

Now choose variables $(x, \xi)$ as in the proof of Lemma 1 , but so as to have $0<\mu_{i}<\varepsilon$ for $i=1, \ldots, n-1$ (see (8)); if necessary, a change of variables $x^{\prime}=T x$ can be used to bring this about.

We modify the reasoning used in Lemma 1, by writing now

$$
f(z(x, \xi))=F_{0} u+f^{(2)}(x, \xi, u)
$$

where $F_{0}=F(0)=\left(\partial f_{i}(0,0) / \partial u_{j}\right)_{i, j=1, \ldots, p}$. We also write, for $i=1, \ldots, n$,

$$
\begin{aligned}
X_{i}^{(2)}(x, \xi) & =X_{i}(x, \xi)-\sum_{j=1}^{n} \frac{\partial X_{i}}{\partial x_{j}}(0,0) x_{j} \\
& = \begin{cases}X_{i}(x, \xi)-\lambda_{i} x_{i}-\mu_{i} x_{i+1}, & i=1, \ldots, n-1, \\
X_{n}(x, \xi)-\lambda_{n} x_{n}, \quad i=n,\end{cases}
\end{aligned}
$$

and

$$
X^{(2)}=\sum_{i=1}^{n} X_{i}^{(2)}(x, \xi) \frac{\partial}{\partial x_{i}}
$$


Clearly we have

$$
\begin{aligned}
& \frac{\partial f_{i}^{(2)}}{\partial u_{j}}(0,0,0)=0, \quad i, j=1, \ldots, p, \\
& X_{i}^{(2)}(0, \xi)=0, \quad i=1, \ldots, n, \quad \text { and } \\
& \frac{\partial X_{i}^{(2)}}{\partial x_{j}}(0,0)=0, \\
& i, j=1, \ldots, n \text {, }
\end{aligned}
$$

and (1) may be rewritten as

$$
\left\{\sum_{i=1}^{n} \lambda_{i} x_{i} \frac{\partial}{\partial x_{i}}+\sum_{i=1}^{n-1} \mu_{i} x_{i+1} \frac{\partial}{\partial x_{i}}-F_{0}\right\} u(x, \xi)=v(x, \xi)
$$

where $v(x, \xi)$ is defined by

$$
v(x, \xi)=f^{(2)}(x, \xi, u(x, \xi))-\left(X^{(2)}+Y\right) u(x, \xi) .
$$

For any $i=1, \ldots, n-1$ and any $\alpha \in \mathscr{G}_{n}$ with $\alpha_{i+1}>0$, we denote by $\alpha^{(i)}$ the element of $g_{n}$ obtained by increasing $\alpha_{i}$ by 1 and decreasing $\alpha_{i+1}$ by 1 ; if $\alpha_{i+1}=0$ we define $\alpha^{(i)}=0$, recalling that $u_{0}(\xi)=0$ since ord $u(z)>k>1$. Then equating the coefficients of $x^{\alpha}$ on either side of (19) gives, for all $\alpha \in \mathscr{I}_{n}$,

$$
\left\{(\lambda \cdot \alpha) I-F_{0}\right\} u_{\alpha}(\xi)+\sum_{i=1}^{n-1} \mu_{i}\left(\alpha_{i}+1\right) u_{\alpha}^{(i)}(\xi)=v_{\alpha}(\xi)
$$

We deduce at once from (21) the relation

$$
\left|u_{\alpha}\right|(\xi)<\frac{1}{c_{0}|\alpha|} M\left\{\left|v_{\alpha}\right|(\xi)+\sum_{i=1}^{n-1} \mu_{i}\left(\alpha_{i}+1\right)\left|u_{\alpha^{(i)}}\right|(\xi)\right\}
$$

which is valid for all $\alpha \in g_{n}$ with $|\alpha|>k$, from which we get at once

$$
\left|u_{\alpha}\right|(\xi)<\frac{1}{c_{0}|\alpha|} M\left\{\left|v_{\alpha}\right|(\xi)+2 \varepsilon|\alpha| \sum_{i=1}^{n-1}\left|u_{\alpha^{(i)}}\right|(\xi)\right\}
$$

for $|\alpha|>k$. On the other hand, $\left|v_{\alpha}\right|(\xi)$ is the coefficient of $x^{\alpha}$ in the expansion of $|v|(x, \xi)$, and, if we write

$$
\left|X^{(2)}\right|=\sum_{i=1}^{n}\left|X_{i}^{(2)}\right|(x, \xi) \frac{\partial}{\partial x_{i}} \quad \text { and } \quad|Y|=\sum_{j=1}^{s}\left|Y_{j}\right|(x, \xi) \frac{\partial}{\partial \xi_{j}}
$$

we have

$$
|v|(x, \xi)<\left|f^{(2)}\right|(x, \xi,|u|(x, \xi))+\left\{\left|X^{(2)}\right|+|Y|\right\}|u|(x, \xi) .
$$

We stop to note here that by virtue of (18) we may write

$$
\begin{aligned}
& \left|X_{i}^{(2)}\right|(x, \xi)=\sum_{j=1}^{n} b_{i j}(x, \xi) x_{j} \quad \text { for } i=1, \ldots, n \\
& \quad \text { where } b_{i j}(x, \xi) \in \mathbf{C}\{x, \xi\}_{+} \text {and } b_{i j}(0,0)=0, i, j=1, \ldots, n ;
\end{aligned}
$$


furthermore, we have

$$
\left|Y_{j}\right|(0, \xi)=0 \text { for } j=1, \ldots, s
$$

and, as a consequence of (18),

$$
\frac{\partial\left|f_{i}^{(2)}\right|}{\partial u_{j}}(0,0,0)=0 \text { for } i, j=1, \ldots, p \text {. }
$$

Let us now agree that whenever $y(x, \omega)$ is a formal power series in $x=\left(x_{1}, \ldots, x_{n}\right)$ and some other set of variables $\omega=\left(\omega_{1}, \ldots, \omega_{r}\right)$ (we have only two cases in mind: (i) $\omega=\xi$ and $r=s$; and (ii) $\omega=(\xi, u)$ and $r=s+$ $p$ ) then $y^{\sharp}(t, \omega)$ denotes the element of $\left.\mathrm{C}[t, \omega]\right]$ obtained by substituting $x_{1}=x_{2}=\cdots=x_{n}=t$ into $y(x, \omega)$; similarly, if

$$
u(x, \omega)=\left(u_{1}(x, \omega), \ldots, u_{p}(x, \omega)\right) \text {, }
$$

then

$$
u^{\sharp}(t, \omega)=\left(u_{1}^{\#}(t, \omega), \ldots, u_{p}^{\#}(t, \omega)\right) \text {. }
$$

Thus, for example, if $y(x, \xi)=\Sigma_{\alpha \in g_{n}} y_{\alpha}(\xi) x^{\alpha}$, then $y^{\sharp}(t, \xi)=\Sigma_{l=0}^{\infty} y_{(h)}^{\#}(\xi) t^{l}$ where, for each $l=0,1,2, \ldots y_{(b)}^{\sharp}(\xi)=\Sigma_{|\alpha|-l} y_{\alpha}(\xi)$. We observe that if, in fact, $y(x, \xi) \in \mathrm{C}[x, \xi]]_{+}$, then, for $i=1, \ldots, n$,

$$
\left(\frac{\partial y}{\partial x_{i}}\right)^{\sharp}(t, \xi) \prec \frac{\partial y^{\sharp}}{\partial t}(t, \xi) \text {. }
$$

To see this, let $\delta^{(i)}$ denote the element $(0,0, \ldots, 0,1,0, \ldots, 0)$ of $\mathscr{G}_{n}$ (i.e., 1 in the $i$ th place), and suppose

$$
y(x, \xi)=\sum_{\alpha \in g_{n}} y_{\alpha}(\xi) x^{\alpha} .
$$

Then

$$
\frac{\partial y}{\partial x_{i}}(x, \xi)=\sum_{\alpha \in \mathcal{G}_{n}}\left(\alpha_{i}+1\right) y_{\alpha+\delta^{(i)}}(\xi) x^{\alpha}
$$

so, for each $l=0,1,2, \ldots$,

$$
\begin{aligned}
\left(\frac{\partial y}{\partial x_{i}}\right)_{(l)}^{\#}(\xi) & =\sum_{|\alpha|=l}\left(\alpha_{i}+1\right) y_{\alpha+\delta^{(i)}}(\xi) \prec(l+1) \sum_{|\beta|=l+1} y_{\beta}(\xi) \\
& =(l+1) y_{(l+1)}^{\#}(\xi) .
\end{aligned}
$$

Since this last expression is the coefficient of $t^{l}$ in $\partial y^{\sharp}(t, \xi) / \partial t,(27)$ is established. Applying it to (23), we deduce that

$$
\begin{aligned}
|v|^{\sharp}(t, \xi)< & \left|f^{(2)}\right|^{\sharp}\left(t, \xi,|u|^{\#}(t, \xi)\right) \\
& +b(t, \xi) t \frac{\partial|u|^{\sharp}(t, \xi)}{\partial t}+\sum_{j=1}^{s}\left|Y_{j}\right|^{\sharp}(t, \xi) \frac{\partial|u|^{*}(t, \xi)}{\partial \xi_{j}}
\end{aligned}
$$


where we have written $b(t, \xi)$ for $\sum_{i, j=1}^{n} b_{i j}^{\#}(t, \xi)$. On the other hand, fixing $l>k$, and summing in (22) over those $\alpha \in \mathscr{G}_{n}$ with $|\alpha|=l$ gives

$$
l|u|_{(l)}^{\#}(\xi) \prec \frac{1}{c_{0}} M\left\{|v|_{(l)}^{\#}(\xi)+2 \varepsilon l \sum_{i=1}^{n-1} \sum_{|\alpha|=l}\left|u_{\alpha^{(i)}}\right|(\xi)\right\},
$$

and so, clearly,

$$
l|u|_{(l)}^{\#}(\xi) \prec \frac{1}{c_{0}} M\left\{|v|_{(l)}^{\#}(\xi)+2 \varepsilon l(n-1)|u|_{(l)}^{\#}(\xi)\right\} .
$$

Since $|u|_{(\ell)}^{*}(\xi)=0$ for $l<k$, by hypothesis, we deduce at once that

$$
t \frac{\partial|u|^{\sharp}}{\partial t}(t, \xi) \prec \frac{1}{c_{0}} M\left\{|v|^{\#}(t, \xi)+2 \varepsilon(n-1) t \frac{\partial|u|^{\#}}{\partial t}(t, \xi)\right\} .
$$

Combining this last relation with (28) yields

$$
t \frac{\partial|u|^{\sharp}}{\partial t}(t, \xi) \prec A(t, \xi)\left\{t \frac{\partial|u|^{\sharp}}{\partial t}(t, \xi)\right\}+h\left(t, \xi,|u|^{\sharp}(t, \xi),\left\{\frac{\partial|u|_{i}^{\#}}{\partial \xi_{j}}(t, \xi)\right\}\right)
$$

where $A(t, \xi)=\left(2 \varepsilon(n-1) / c_{0}\right) M+b(t, \xi) I$ and

$$
h(t, \xi, v, q)=\frac{1}{c_{0}} M\left\{\left|f^{(2)}\right|^{\sharp}(t, \xi, v)+\sum_{j=1}^{s}\left|Y_{j}\right|^{\sharp}(t, \xi) q_{(j)}\right\} ;
$$

here $q_{(j)}$ denotes the $p$-tuple $\left(q_{1 j}, q_{2 j}, \ldots, q_{p j}\right)$ for $j=1, \ldots, s$. We note first that $A(t, \xi)$ is convergent, and, because of (16) and (24), satisfies the hypothesis of Lemma 3. Moreover, by virtue of (25) and (26), $h(t, \xi, v, q)$ satisfies the hypotheses of Lemma 4. Applying Lemma 3 first, we may, in effect, suppose that $A(t, \xi)=0$. Lemma 4 then applies to show that $|u|^{*}(t, \xi)$ is convergent. From this it follows trivially that $|u|(x, \xi)$ and therefore $u(z)$ are convergent, which was to be shown.

Proof of Theorem 1 (CONCluded). We suppose first that $k_{0}>1$. If $\psi \in\left[A_{k_{0} 0}\right]^{p}$ satisfies $\Phi_{k_{0}}(\psi)=0$, and if $v(z)$ is chosen in $\left.[C[z]]_{0}\right]^{p}$ so as to satisfy $[v(z)]_{k_{0}}=\psi$, then

Thus, defining

$$
\operatorname{ord}_{s}\{f(z, v(z))-X v(z)\}>k_{0} .
$$

$$
\tilde{f}(z, u)=f(z, v(z)+u)-X v(z) \in\left[C\left[[z, u]_{0}\right]^{p},\right.
$$

we have $\operatorname{ord}_{s} \tilde{f}(z, 0)>k_{0}$. Therefore, by Lemma 1 , there exists $w(z) \in$ $\left.[C[z]]_{0}\right]^{p}$ with ord $s w(z)>k_{0}$ and $X w(z)=\tilde{f}(z, w(z))$. It follows that $u(z)=$ $v(z)+w(z)$ satisfies $[u(z)]_{k_{0}}=\psi$ and (1); moreover, the uniqueness of this solution $u(z)$ of (1), with $[u(z)]_{k_{0}}=\psi$ follows from the corollary to Lemma 2 (see the remark following the proof of that corollary) as does assertion (i) of Theorem 1. To prove assertion (ii) it suffices to note that, if $\psi$ is convergent, 
in the above argument we may choose $v(z)$ in $\left[\mathbf{C}\{z\}_{0}\right]^{p}$ so that $\tilde{f}(z, u) \in$ $\left[\mathbf{C}\{z, u\}_{0}\right]^{p}$ and Lemma 5 then asserts that $w(z) \in\left[\mathbf{C}\{z\}_{0}\right]^{p}$; this means that $u(z)=v(z)+w(z)$ is convergent as well.

Finally, we treat the case $k_{0}=0$, as follows: Choose any variables $(x, \xi)$ adapted to $S$. Since the matrix

$$
F_{0}=\left(\frac{\partial f_{i}}{\partial u_{j}}(0,0)\right)_{i, j=1, \ldots, p}
$$

is now assumed to be invertible the implicit function theorem asserts the existence of a unique solution $\left.u_{0}(\xi) \in[\mathrm{C}[\xi]]_{0}\right]^{p}$ of the equation $f\left(z(0, \xi), u_{0}(\xi)\right)$ $=0$; in fact, $u_{0}(\xi)$ is even convergent. We now define $\psi \in\left[A_{1,0}\right]^{p}$ so that $\psi=u_{0}(\xi)$ in the $(x, \xi)$ variables. Clearly, $\Phi_{1}(\psi)=0$, so, by the part of Theorem 1 already proved, there is a unique solution $\left.u(z) \in[\mathrm{C}[z]]_{0}\right]^{p}$ of (1) with $[u(z)]_{1}=\psi$, and, in fact, $u(z) \in\left[\mathbf{C}\{z\}_{0}\right\}^{p}$. On the other hand, given any solution $u^{*}(z) \in\left[C[z]_{0}\right]^{p}$ of $(1)$, we must have $f\left(z(0, \xi), u^{*}(z(0, \xi))\right)=0$, so $u^{*}(z(0, \xi))=u_{0}(\xi)$, i.e., $\left[u^{*}(z)\right]_{1}=\psi$, and therefore $u^{*}(z)=u(z)$. Our proof is now complete.

\section{REFERENCES}

1. M. Artin, On the solutions of analytic equations, Invent. Math. 5 (1968), 277-291.

2. N. Dunford and J. T. Schwartz, Linear operators. Part II, Interscience, New York, 1963.

3. F. R. Gantmacher, The theory of matrices. Vol. 2, Chelsea, New York.

4. E. Goursat, Lecons sur l'intégration des équations aux dérivées partielles du premier ordre, A. Hermann, Librairie Scientifique, Paris, 1891.

5. H. Poincaré, Sur les propriétés des fonctions définies par les équations aux différences partielles, Oeuvres, Tome 1. Gauthier-Villars, Paris, 1951.

Department of Mathematics, City College of New York, New York, New York 10031

Graduate School and Universtty Center (CUNY), New York, New York 10036 